\title{
CP Violation and Leptogenesis
}

\author{
Andy Acker $^{a)}$, Hisashi Kikuchi ${ }^{a)},{\text { Ernest } \mathrm{Ma}^{a)} \text { and Utpal Sarkar }}^{b)}$ \\ a) Physics Department, University of California, Riverside, CA 92521, \\ USA. \\ b) Theory Group, Physical Research Laboratory, Ahmedabad-380009, India.
}

\begin{abstract}
Recently a model of chaotic inflation was proposed, where the right handed sneutrinos drive the baryogenesis. We study some of the details of the model, particularly the aspect of $\mathrm{CP}$ violation, and determine the number of right handed sneutrinos required for the viability of such models.
\end{abstract}

UCRHEP-T109 
Recently a model has been proposed [1], where the inflation is driven by the superpartner of the right handed neutrino. Although the inflationary scenario is quite appealing for the solution of the horizon and the flatness problem [2], it is difficult to formulate a particle physics model consistently. In addition to solving this problem, this model generates (B-L) asymmetry at lower temperature in the post inflationary universe through violation of lepton number. This model requires the right handed neutrinos which are singlets under all groups for one of these fields to be the inflaton. The Majorana mass terms for these fields have been introduced in this model by hand to explain the COBE result [3], but otherwise there is no natural reason for the origin of that scale in the model.

The main objective of this article is to study the $\mathrm{CP}$ violation in these models, determine the number of singlet fields required for $\mathrm{CP}$ violation and count the number of $\mathrm{CP}$-violating phases which can be present in the combination of the Yukawa couplings appearing in the processes under consideration [4. We shall also give a natural explanation for the origin of the Majorana mass scale. We consider the particle content to be exactly same as that of ref 1 , with the exception of the number of right handed neutrinos $\left(N_{\alpha}\right.$ 's). We start with $\bar{n}$ number of these scalars $\left(N_{\alpha}, \quad \alpha=1, \ldots, \bar{n}\right)$ all of which carry a (B-L) number -1 . While any of the $N_{\alpha}$ can serve as the inflaton, for the time being let us consider the scalar partner of $N_{1}$ to be the inflaton. We do not give explicit mass to these fields. The only mass scales in this model are the grand unified symmetry breaking scale $M_{u} \sim\langle\Sigma\rangle$, where $\Sigma$ is a 24-plet of SU(5) and breaks the SU(5) symmetry, and $M_{w} \sim\left\langle H_{u}\right\rangle \sim\left\langle H_{d}\right\rangle$, where $H_{u}$ and $H_{d}$ are $5-$ and $\overline{5}$-plets of $\mathrm{SU}(5)$ and mediates the standard model symmetry breaking and give masses to the fermions. We also assume that the singlet fields have odd R-parity like other fermions.

The Yukawa part of the superpotential is given by,

$$
W=h^{\alpha j} N_{\alpha}^{c} l_{j} H_{u}+k^{i j} e_{i}^{c} l_{j} H_{d}
$$

where, $\alpha, \beta=1, \ldots, \bar{n}$ are the indices for the fields $N_{\alpha}$ and $i, j=1, \ldots, n$ are the indices for the left handed leptons. We assume that (B-L) is a global symmetry of the superpotential, hence higher order terms involving the $N_{\alpha}$ 's are forbidden. Although finally we are interested in $n=3$, for our analysis we shall take $n$ to be arbitrary.

Until the time $H \sim M_{\alpha \beta}$ the $\tilde{N}_{1}$ field will roll down the potential and after that oscillate around its minimum, $\tilde{N}_{1}=0$, with frequency $M$. Once 
the universe cools down to about $10^{4} \mathrm{GeV}$, the coherent oscillation starts to decay into lighter particles thus reheating the Universe again to about $10^{10}$ $\mathrm{GeV}$. During this period of reheating the decay of $\tilde{N}_{1}$ field produces (B-L) number asymmetry through lepton number violation due to $\mathrm{CP}$ violation. This (B-L) asymmetry remains as a baryon number asymmetry after the electroweak anomalous processes $[5]$.

While the origin of the $N_{\alpha}$ mass term is not critical to our discussion, we would like to suggest an interesting possibility. After the field $\Sigma$ acquires a vev, there will be new gravity induced nonrenormalizable contributions to the Yukawa coupling, which are not protected by any symmetry. Such terms have been considered in the literature to solve the bad fermion mass relations in SU(5) GUT and some other contexts [6]. We are interested in the term which involves the fields $N_{\alpha}$, this is given by,

$$
W=\frac{1}{M_{P}} H_{\alpha \beta} N_{\alpha} N_{\beta} \Sigma \Sigma .
$$

When the field $\Sigma$ acquire a vev, namely, $\langle\Sigma\rangle \sim M_{u} \sim 10^{16} \mathrm{GeV}$ (for supersymmetric SU(5) GUT[7]), this term will induce a lepton number violating mass term for the fields $N_{i}$ of the right magnitude $\sim \frac{M_{u}{ }^{2}}{M_{P}} H_{\alpha \beta} \sim M_{\alpha \beta} \sim 10^{13}$ $\mathrm{GeV}$ to be consistent with the COBE data [B], $\delta T / T \sim 10^{-5}$.

In the generation of the (B-L) asymmetry through the lepton number asymmetry [1, 5, 9, 10], the CP violation plays a crucial role [11], and we now study systematically this question of $\mathrm{CP}$ violation and the decay of the heavy neutrinos in a general way, trying to determine the number of phases present in the Yukawa type couplings. The $\mathrm{CP}$ violating parameter $\epsilon$, which is the asymmetry in the $\tilde{N}_{\alpha}$ decay between that into leptons $l_{i} \tilde{H}_{u}$ and that into antisleptons $\tilde{l}_{i}^{*} H_{u}^{*}$, is defined as

$$
\epsilon=\frac{\left|A\left(\tilde{N}_{\alpha} \rightarrow l_{i} \tilde{H}_{u}\right)\right|^{2}-\left|A\left(\tilde{N}_{\alpha} \rightarrow \tilde{l}_{i}^{*} H_{u}^{*}\right)\right|^{2}}{|A|^{2}}
$$

and is given by (see Fig.1 of ref. [1]),

$$
\epsilon=\frac{\ln 2}{8 \pi} \frac{\operatorname{Im}\left(h_{\alpha i} h_{\alpha j} h_{\beta i}^{*} h_{\beta j}^{*}\right)}{h_{\alpha i} h_{\alpha i}^{*}}=\frac{\ln 2}{8 \pi} \frac{T_{\alpha \beta i j}}{\left|h_{\alpha i}\right|^{2}}
$$

with $t_{\alpha \beta i j}=\left(h_{\alpha i} h_{\alpha j} h_{\beta i}^{*} h_{\beta j}^{*}\right)$ and $T_{\alpha \beta i j}=\operatorname{Im}\left(t_{\alpha \beta i j}\right)$. While this expression is clearly invariant under rephasing of the fields $l_{i}$ [10], the invariance of 
$\epsilon$ under the transformation $N_{\alpha} \rightarrow e^{i \delta_{\alpha}} N_{\alpha}$ is not obvious. Indeed, under such a transformation the quantity $t_{\alpha \beta i j} \rightarrow e^{2 i\left(\delta_{\alpha}-\delta_{\beta}\right)} t_{\alpha \beta i j}$. It would appear that a suitable choice of the phases $\delta_{\alpha}-\delta_{\beta}$ can make $t_{\alpha \beta i j}$ real, and thus give $\epsilon=0$. However, rephasing of the fields $N_{\alpha}$ introduces new phases in the propagator and wave function through complex phases in the mass parameter which exactly cancel the extra phase picked up by $t_{\alpha \beta i j}$, leaving $\epsilon$ invariant. In the leptogenesis models we need only consider the effect of $\mathrm{CP}$ violation in the decay of heavy neutrinos at temperatures well above the electro-weak symmetry breaking scale, where the Higgs field has not acquired a vev, and the left handed neutrinos are massless. We thus choose to work in the basis where $N_{\alpha}$ are the real, positive mass eigenstates of the right handed neutrinos, and note that this choice exhausts our freedom to make phase transformations on the fields $N_{\alpha}$.

The CP violating parameter describing the asymmetry in the decay of Majorana neutrinos $N_{\alpha}$ into leptons vs. antileptons is proportional to the same combination of Higgs-Yukawa couplings $T_{\alpha \beta i j}$ appearing in Eq. 4 (see ref. [10). We wish to determine under what conditions $T$ is non-zero, and in general, find the number of independent $T$ 's which can appear in the asymmetry parameter. From the definition of the $T$ 's, one can immediately get the following relations,

$$
T_{\alpha \beta i j}=T_{\alpha \beta j i}=-T_{\beta \alpha i j}=-T_{\beta \alpha j i}
$$

i.e., the $T$ is antisymmetric under the interchange of the heavy singlet indices, while it is symmetric under the interchange of the $\nu_{i}$ 's. Thus, for $\bar{n}=1$, i.e., only one generation of heavy singlet field $N_{\alpha}$ there is no CP violation. This relation, however, does not determine the minimum number neutrinos required for $\mathrm{CP}$ violation.

We now proceed to calculate the number of phases that can appear in $T_{\alpha \beta i j}$ when $n$, the number of left handed neutrinos, and $\bar{n}$, the number of right handed neutrinos, are arbitrary. We find that the maximum number of phases which can occur is equal to the number of independent phase in the $\bar{n} \times \bar{n}$ hermitian matrix $H$

$$
H_{\alpha \beta}=h_{\alpha j} h_{\beta j}^{*}=\left(h h^{\dagger}\right)_{\alpha \beta},
$$

where $h_{\alpha j}$ is the Yukawa coupling between $N_{\alpha}$ and $l_{j}$. To see this recall that the left handed neutrinos are massless, and hence degenerate. Therefore, 
there are no observable consequences to performing a unitary transformation on the fields $l_{i}$; in particular, the magnitude of $\mathrm{CP}$ violation in the decay of $N_{\alpha}$ must be invariant under such transformations. Inspection of Eq. 4 reveals that $H_{\alpha \beta}$ is the minimum set of Yukawa couplings appearing in the $\mathrm{CP}$ violation parameter which is invariant under this transformation. It should be noted that unitary transformations of the $l_{i}$ leave the structure of the Standard Model lagrangian unchanged.

If $n \geq \bar{n} H$ is an unconstrained $\bar{n} \times \bar{n}$ Hermitian matrix, whereas if $n<\bar{n}$ there are additional constraints the number of independent elements in $H$. We find that the number of independent complex phases in $H$ is given by

$$
N_{\text {phases }}= \begin{cases}\frac{\bar{n}(\bar{n}-1)}{2} & n \geq \bar{n} \\ n \bar{n}-\frac{n(n+1)}{2} & n<\bar{n}\end{cases}
$$

For the case of $n \geq \bar{n}$ its possible to choose a basis where the matrix of Higgs Yukawa couplings $h_{\alpha i}$ can itself be written as an $\bar{n} \times \bar{n}$ hermitian matrix. To see this explicitly note that for $n \geq \bar{n}$ we can construct a unitary matrix $V_{j k}$ such that

$$
h^{\alpha j} V_{j k}=0 \text { for } k>\bar{n} .
$$

By performing the unitary transformation on the lepton doublet $l_{j}, l_{k}=$ $V_{k j}^{-1} l_{j}$ it is clear that only the $l_{k}$ with $k \leq \bar{n}$ couple to the right-handed singlet fields $N_{\alpha}$. In this basis the couplings are completely described by an $\bar{n} \times \bar{n}$ square matrix $h^{\prime}$. Finally, recall that any square matrix $h^{\prime}$ can be written as the product $h^{\prime}=h W$, where $W$ is unitary and $\mathrm{h}$ is hermitian. Thus by again performing a unitary transformation on the lepton doublets $l_{k}$, the Higgs Yukawa coupling becomes

$$
h^{\alpha j} N_{\alpha}^{c} l_{j} H_{u}
$$

where, as advertised, $h$ is an $\bar{n} \times \bar{n}$ hermitian matrix.

We may use the fact that $h$ is hermitian to show how the complex phases manifest themselves in the quantity $T_{\alpha \beta i j}$, and do this explicitly for $\bar{n}=2$ and 3. (If $\bar{n}=1, h$ is real and $T$ vanishes identically, hence, as discussed above there is no CP violation). For $\bar{n}=2, h$ contains one phase, and there

\footnotetext{
${ }^{1}$ This is the same number of phases that can occur in an $\bar{n} \times \bar{n}$ Majorana mass matrix [4, however; the physical context here is quite different.
} 
are three distinct $T$ 's: $T_{1211}, T_{1212}$ and $T_{1222}$. These are related to each other by

$$
\frac{T_{1211}}{\left|t_{1211}\right|}=\frac{T_{1212}}{\left|t_{1212}\right|}=\frac{T_{1222}}{\left|t_{1222}\right|}=\sin \left(2 \delta_{12}\right)
$$

where $\delta_{\alpha j}$ is defined by $h^{\alpha j}=\left|h^{\alpha j}\right| e^{i \delta_{\alpha j}}$. For $\bar{n}=3$ there are 18 distinct $T$ 's, however, there are only 3 independent phases, appearing in 9 possible combinations. These are summarized by the following three relations

$$
\begin{gathered}
\frac{T_{\alpha \beta \alpha \alpha}}{\left|t_{\alpha \beta \alpha \alpha}\right|}=\frac{T_{\alpha \beta \beta \beta}}{\left|t_{\alpha \beta \beta \beta}\right|}=\frac{T_{\alpha \beta \alpha \beta}}{\left|t_{\alpha \beta \alpha \beta}\right|}=\sin \left(2 \delta_{\alpha \beta}\right) \\
\frac{T_{\alpha \beta \alpha \gamma}}{\left|t_{\alpha \beta \alpha \gamma}\right|}=\frac{T_{\alpha \beta \beta \gamma}}{\left|t_{\alpha \beta \beta \gamma}\right|}=\sin \left(\delta_{\alpha \beta}+\delta_{\alpha \gamma}-\delta_{\beta \gamma}\right) \\
\frac{T_{\alpha \beta \gamma \gamma}}{\left|t_{\alpha \beta \gamma \gamma}\right|}=\sin \left(2 \delta_{\alpha \gamma}-2 \delta_{\beta \gamma}\right)
\end{gathered}
$$

where $\alpha<\beta$ and $\gamma \neq \alpha \neq \beta$. In this notation the three independent phases appearing in $T_{\alpha \beta i j}$ are $\delta_{12}, \delta_{13}$ and $\delta_{23}$.

In conclusion we have examined leptogenesis models where the initial lepton number asymmetry is generated by a CP asymmetry in the decay of a heavy neutrino(sneutrino) into leptons with respect to the decay into antileptons(sleptons). The Higgs Yukawa couplings $h_{\alpha i}$ that gives rise to these decays must contain at least one complex phase. We have calculated the number of complex phases that can occur in this coupling for an arbitrary number of heavy right handed neutrinos and massless left handed neutrinos. We find that, for $\bar{n}$ heavy right handed neutrinos and $n$ left handed neutrinos the number of independent phases appearing in the asymmetry parameter $\epsilon$ is given by equation (7). Thus a minimum of 2 right handed neutrinos(sneutrinos) and one left handed neutrino are required for CP violation to occur in right handed neutrino decay. For the 3 generation case $(n=\bar{n}=3)$ there are three independent phases in the Higgs Yukawa couplings. Finally, we note that this result is qualitatively different from CP violation in charged-current weak interactions arising from a complex phase in the Kobayashi-Maskawa matrix.

\section{Acknowledgements}

Two of us (U.S. and A.A.) wish to thank the Department of Physics at the University of California, Riverside for their hospitality. A.A. also wishes to 
thank the Australian Department of Immigration for making possible the trip to UCR. This work was supported in part by Department of Energy contract number DE-AT03-87ER40327. 


\section{References}

[1] H. Murayama, H. Suzuki, T. Yanagida and J. Yokoyama, Phys. Rev. Lett. 70 (1993) 1912.

[2] A.H. Guth, Phys. Rev. D 23 (1981) 347.

[3] G.F. Smoot et al., Astrophys. J. Lett. 396 (1992) L1.

[4] M. Doi, T. Kotani, H. Nishiura, K.Okuda and E. Takasugi, Phys. Lett. B 102 (1981) 323; J. Schechter and J.W.F. Valle, Phys. Rev. D 25 (1982) 774; B. Kayser, Phys. Rev. D 30 (1984) 1023; J.F. Nieves and P.B. Pal, Phys. Rev. D 36 (1987) 315.

[5] V.A. Kuzmin, V.A Rubakov and M.E. Shaposhnikov, Phys. Lett. B 155 (1985) 36.

[6] C.T. Hill, Phys. Lett. B 135 (1984), 47; C. Wetterich, Phys. Lett. B 110 (1982), 384; J. Ellis and M.K. Gaillard, Phys. Lett. B 88 (1979), 315; Q. Shafi and C. Wetterich, Phys. Rev. Lett. 52 (1984), 875; M.K. Parida, P.K. Patra and A.K. Mohanty, Phys. Rev. D 39 (1989), 316; B. Brahmachari, P.K. Patra, U. Sarkar and K. Sridhar, Int. Jour. Mod. Phys. A (to appear).

[7] See for example, A. Masiero in "Grand Unification with and without Supersymmetry and Cosmological Implications", International School for Advanced Studies Lecture Series No. 2, published by World Scientific, (1984), p.1; see also A. Zee (ed.) "Unity of Forces in the Universe", Vol. 1, published by World Scientific, 1982.

[8] D.S. Salopeck, Phys. Rev. Lett. 69 (1992) 247.

[9] M. Fukugita and T. Yanagida, Phys. Lett. B 174 (1986) 45.

[10] M.A. Luty, Phys. Rev. D 45 (1992) 445.

[11] A.D. Sakharov, JETP Letters 5 (1967) 24. 